\title{
Les débats médiatiques télévisé Une hétérogénéité de classement
}

The televised media debates A heterogeneity of classification

Received: 20/11/2019 ; Accepted: 09/12/2019

\section{Résumé}

En nous basant sur l'analyse des interactions verbales dans les débats médiatiques télévisés, nous cherchons à mettre en exergue les contraintes situationnelles et discursives qui régissent leur déroulement et donc détermine leur classement que ça soit : au niveau de la typologie des interactions ou au niveau du genre de l'oral. Cette analyse vise à mettre en évidence la complexité de la relation construite entre les sujets parlant qui partagent incessamment une pluralité de positionnement due aux rôles interlocutifs et interactionnels occupés. Ce qui aboutit à une diversité de mode d'allocution adopté.

Mots clés: Relation interpersonnelle, rôle interlocutif, rôle interactionnel, symétrie, coopération.
Souheila Ziada*

Université Frères

Mentouri Constantine1

\section{Abstract}

Based on the analysis of verbal interactions in television media debates, we seek to highlight the situational and discursive constraints that govern their unfolding and this determine their ranking what so ever: level of the type of interactions or at the level of the oral genre. This analysis aims to highlight the complexity of the relationship built between talking subjects who share a plurality of positioning due to the busy interlocutoral and interactional roles. This leads to a diversity of methods of speaking

Keywords: Interpersonal relationship, interpersonal role, interactional role, symmetry, cooperation..

\footnotetext{
* Corresponding author, e-mail: souheilaziada.ghanem@gmail.com
} 


\section{Introduction :}

Tout comme les productions écrites, les productions orales peuvent être aussi classées en type et genre. Selon les interactionnistes le type est étroitement liés aux événements de la communication, c'est-à-dire, il se détermine à travers des circonstances externes, car il « se fond sur des données relatives à la situation: participants, cadre (lieu et temps), objectif $»^{(1)}$. Ces contraintes situationnelles régissent le déroulement des interactions verbales, notamment, d'une part la constitution de la relation interpersonnelle qui peut être symétrique ou complémentaire et coopérative ou conflictuelle ; et d'autre part l'activité discursive qui détermine le genre oral de l'interaction, à travers l'organisation des échanges et le choix du matériel linguistique adéquat. Ainsi, nous pouvons parler, par exemple, des genres de discours : argumentatif qui est fréquent dans les débats, explicatif qui caractérise les interactions verbales en situation d'apprentissage, exhortatif dans les mises en scènes publicitaire, etc...

En ce qui concerne cette présente étude, nous nous sommes intéressée à l'analyse des interactions verbales dans les débats médiatiques télévisés et plus précisément les émissions "Face à face » de France 24 d'expression française pour dégager leurs spécificités avec toutes les contraintes situationnelles qui régissent leur développement et influencent la relation interpersonnelle qui s'instaurent entre les sujets parlant et donc caractérisent le mode d'allocution adopté et déterminent leurs classement que ça soit : au niveau de la typologie des interactions ou au niveau du genre de l'oral. Pour atteindre notre objectif, nous nous sommes posée les questions suivantes : quelles places institutionnelles et discursives occupent les sujets qui communiquent dans les débats médiatiques télévisés, surtout que le cadre participatif est complexe mettant en présence trois partenaires ? $\mathrm{Ce}$ positionnement aboutit-il à une pluralité de relation interpersonnelle ? Enfin s'il y a une diversité de relation est-ce qu'elle influence le mode de la circulation de la parole ? Pour pouvoir répondre à ce questionnement, nous allons aborder les émissions télévisées «Face à face » qui représentent notre objet d'analyse du point de vue de la typologie et du genre discursivo-interactionnel.

\section{Qu'est-ce qu'un type d'interaction?}

Selon Vion, R. (1992), le type d'interaction dépend aussi bien de la nature des finalités que du degré de formalité. Pour les finalités, il propose deux sortes: les finalités «internes » qui portent sur la qualité du contact avec un seul objectif : la recherche de l'intercompréhension ; et les finalités « externes » qui se traduisent en termes d'action et de modification du réel tel que les consultations médicales, les débats politiques, les enquêtes... Le degré de formalité dépend, quant à lui, du nombre de participants ; de la nature du cadre interactif ; des règles de la circulation de la parole ; du caractère ouvert ou fermé du contrat de la parole; de la nature des rituels d'ouverture et de clôture ; et de la nature des signaux d'écoute. Ces critères de «formalité » et de «finalités» permettent la distinction entre «symétrie/complémentarité», et «coopération/compétition». 
L'interaction est dite symétrique quand l'accent porte sur les efforts pour établir et maintenir l'égalité. Les partenaires donc minimisent leur différence en adoptant un comportement en miroir. Ils jouissent tous des mêmes droits et ont les mêmes obligations. La conversation ordinaire, la discussion, le débat, la dispute en sont des exemples typiques.

L'interaction est dite complémentaire lorsqu'elle se base sur la maximalisation de la différence, où "il y a deux positions différentes possibles. L'un des partenaires occupe une position qui a été désignée comme supérieur, première ou "haute" (one-up), et l'autre la position correspondante dite inférieur, seconde ou "basse" (one-down).» (2)Parmi les interactions complémentaires nous pouvons citer : la consultation médicale, l'enquête, l'entretient, la transaction.

Une interaction est de nature coopérative lorsque les partenaires donnent des marques de bonne volonté et d'entraide lors du déroulement interactionnel, leurs attitudes sont orientées vers des finalités d'intercompréhension. Par contre l'interaction est dite compétitive lorsque les partenaires orientent leurs attitudes vers des finalités de succès, par exemple le désire de séduire, de donner le beau rôle.

Ces relations «symétrique/complémentaire» ou «coopération/compétition » ont un lien très fort avec la progression et le déroulement de l'interaction, dans la mesure où les comportements des interactants (règles de la circulation de la parole, droit à la parole, temps de parole occupé, ...) sont déterminés par la position occupée et l'objectif de la rencontre lors du déroulement interactionnel.

Tous ces paramètres qui régissent le déroulement des interactions verbales font partie de l'ethos collectif. En effet selon Vion, R. ${ }^{(3)}$ "les sujets disposent d'une typologie implicite leur permettant de reconnaître d'entrée le cadre social et institutionnel dans lequel se déroule telle "rencontre » qui les réunit. C'est en associant cette rencontre à un ou plusieurs types d'interaction déjà expérimenté(s) qu'ils peuvent lui donner un sens et donner ainsi du sens à leurs activités et aux énoncés échangés. »

\section{Qu'est-ce qu'un genre discursivo-interactionnel ?}

Le terme « genre » que nous employons dans cette étude renvoie aux différentes activités discursives qui se définissent par : "des critères "internes ». Une argumentation, un récit ou des salutations sont reconnaissables comme tels indépendamment des événements dans lesquels ils s'inscrivent, et qui peuvent être très divers (même si certains types d'activités se rencontrent de façon privilégiée dans certains sites plutôt que dans d'autres). " ${ }^{(4)}$ Ces activités discursives sont étroitement liées d'une part aux matériels linguistiques utilisés, 
et d'autre part à l'organisation des : séquences, des échanges et des actes de langage.

Selon Maingueneau, la langue met au service de ces utilisateurs de nombreux termes et expressions qui peuvent catégoriser leurs échanges communicatifs écrits ou oraux en tant que : description, argumentation, conversation ordinaire, interview,... C'est ainsi qu'il écrit : "Les locuteurs disposent d'une foule de termes pour catégoriser l'immense variété des textes qui sont produits dans une société : conversation, manuel, journal, tragédie, reality-show, roman sentimental, description, polémique, sonnet, récit, maxime, hebdo, tract, rapport de stage, mythe, carte de voux... On notera que la dénomination de ces genres s'appuie sur des critères très hétérogènes.» ${ }^{(5)}$

Ainsi, par exemple en parlant du genre de discours didactique qui est fréquent dans les interactions verbales en situation d'apprentissage, nous allons constater que les activités langagières des interactants se caractérisent par la fréquence des dialogues interrogatifs et donc du couple " question/réponse », ainsi que par les procédés explicatifs tel que les reformulations, les répétitions,... Dans les interactions de commerces, les activités langagières se caractérisent à titre d'exemple par la requête, les remerciements, les salutations ... En effet, selon Virginie André : «(...) les pratiques langagières (c'est-à-dire les productions linguistiques et interactionnelles) actualisées par les interlocuteurs sont appréhendées comme apparaissant dans une situation de communication toujours particulière. Ces pratiques langagières permettent d'accomplir des activités langagières (c'est-à-dire des actions communicatives telles que informer, questionner, remercier, etc.), elles aussi particulières et dépendantes du genre de discours (conversation, réunion de travail, débat, etc.) dans lequel elles sont produites. » ${ }^{(6)}$

\section{Les émissions «Face à face » : quel type et genre discursivo- interactionnel ?}

Les émissions «face à face » qui représentent notre objet d'étude font partie d'un type particulier d'interaction : le débat médiatique que R.Vion définit comme une «interaction qui peut soutenir la comparaison avec la compétition sportive mettant en présence deux sujets.» ${ }^{(7)}$ Ces derniers occupent des places égales du moment qu'ils jouissent des mêmes droits et ont les mêmes obligations. La finalité de leur rencontre est externe, car leur objectif est la persuasion du public autrement dit les téléspectateurs. Ainsi, dans ce type d'interactions, les antagonistes construisent, au niveau des rôles interactionnels, une relation symétrique et compétitive plutôt que coopérative et asymétrique.

Ensuite, le fait qu'il s'agisse de débat, le genre discursivo-interactionel le plus dominant est l'argumentatif car les interactants ont des points de vues le moins qu'on puisse dire divergents. Cette différence de prise de positions se 
fait dans des situations dites «argumentatives» à travers une coordination particulière de leurs actions verbales.

En évoquant la « situation argumentative », Plantin parle de «deux éléments discursifs antagonistes», dont l'un fait référence à l'autre, à propos d'une question qui provoque un désaccord initial à partir duquel émerge toute une interaction argumentative. Cette dernière est définie comme " une situation de confrontation discursive au cours de laquelle sont construites des réponses antagonistes à une question. ${ }^{(8)} \mathrm{La}$ gestion des pratiques langagières dans ce genre discursivo-interactionnel se fait par la manifestation de trois rôles discursifs complémentaires : "Le proposant tient le discours de proposition. L'opposant tient le discours de l'opposition et le tiers prend en charge la question. » $(9)$

Le rôle du Tiers est joué par l'animateur-journaliste qui initie la situation argumentative par une question qui demande des réponses : "Proposition $d u$ Proposant » et "Opposition de l'Opposant ». C'est à lui aussi que revient la gestion de l'interaction au niveau de la circulation de la parole ainsi qu'au niveau des différentes phases de l'interaction comme les reformulations, les récapitulations, ou bien les stabilisations des désaccords.

Les deux autres rôles tenus par les deux débateurs ne sont pas distribués de façon définitive car une redistribution peut surgir à n'importe quel moment en fonction de la technique de sélection du locuteur en place (procédé d'attribution ou auto sélection) ainsi que la question du tiers qui oriente la situation argumentative vers des objectifs prédéfinis.

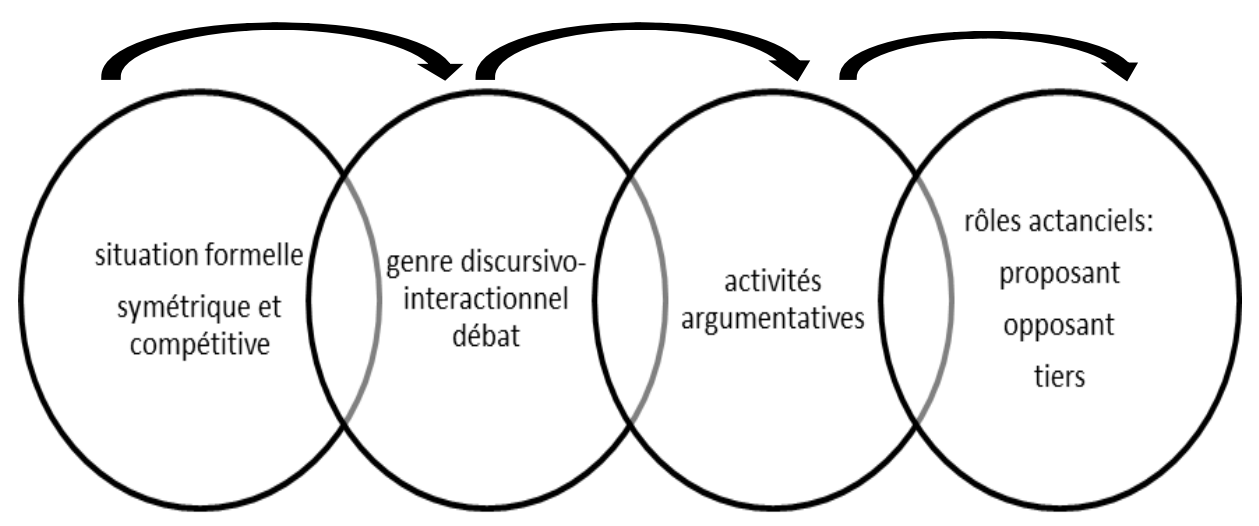

Figure1 : la relation « situation de communication/ type et genre discursivointeractionnel » dans les émissions « face à face » 


\subsection{Le rapport de place :}

Dans les émissions « Face à face », le cadre participatif est plus complexe que celui de la conversation dyadique du fait qu'il met en interaction trois partenaires, l'animateur-journaliste et ces deux invités qui n'occupent pas la même position lors du déroulement interactionnel : que ça soit au niveau de l'activité interlocutive déterminée par la place institutionnelle ou bien au niveau des rôles interactionnels distribués par la place discursive. Par rapport à l'activité interlocutive, il s'agit beaucoup plus de la technique de sélection où l'animateur-journaliste occupe une position haute dans la mesure où il dirige le débat à travers le contrôle de la distribution de la parole ainsi que l'orientation des thèmes. Par rapport aux rôles interactionnels, la position haute est occupée par les deux débatteurs du fait qu'ils sont les plus experts dans le domaine par rapport au thème traité. Ceci implique quatre types de relation : deux d'entre elles se construisent par rapport aux rôles interlocutifs occupés, les deux autres c'est au niveau des activités interactionnelles. Soit le schéma suivant :

Rôles interlocutifs

Animatrice-journaliste

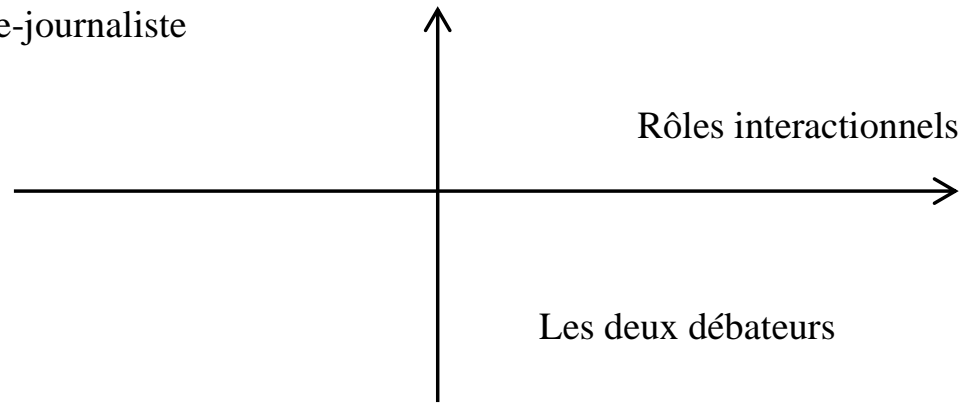

Figure2 : Schémas représentant les positions occupées selon les rôles joués

A partir de ces positions occupées, nous pouvons dire que sur l'axe des rôles interlocutifs deux sortes de relations s'actualisent une asymétrique et complémentaire entre l'animateur-journaliste et ses deux invités, et l'autre symétrique et coopérative entre les deux débatteurs.

Sur l'axe des activités interactionnelles se manifestent aussi deux sorte de relation : une symétrique et compétitive ou conflictuelle entre les deux débatteurs du fait qu'ils jouissent des mêmes droit et ont les mêmes obligations ; et l'autre asymétrique et complémentaire entre les deux débatteurs et l'animateur-journaliste. Soit le schéma suivant : 


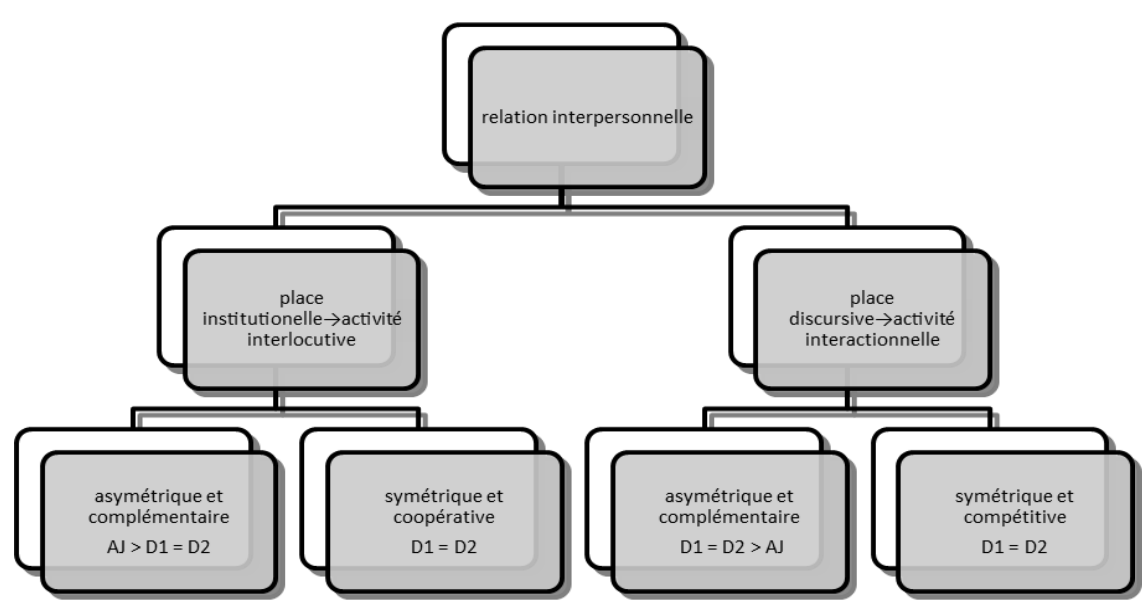

Figure 3 : réseau des relations construites dans les émissions « Face à face ${ }^{(10)}$

\subsection{Le mode d'allocution :}

De par ce rapport de place fluctuant entre les sujets parlants, les interactions verbales de notre corpus mettent successivement deux modes d'allocution : "l'interview » et "la discussion ». En effet, d'une part, pour gérer la progression thématique dans l'interaction, l'animateur-journaliste suit généralement la technique de l'interview en appliquant le mode d'allocution : question/réponse. D'autre part, cette technique n'exclut pas l'aspect de discussion du fait qu'il s'agisse d'une argumentation. Ceci se voit dans les moments où les deux débateurs s'adressent directement l'un à l'autre sans se référer à l'animateur-journaliste. Kerbrat-Orecchioni ${ }^{(11)}$ a écrit à ce sujet: «Le débat teint donc de la discussion (par son caractère argumentatif), et de l'interview (par son caractère médiatique) »

Cette spécificité des interactions verbales dans les débats médiatiques télévisés peut être appréhendée selon le modèle d'analyse en rang de R. Vion qui a ajouté un sixième rang entre l'interaction et la séquence : " le module » qui peut être défini, par exemple, comme un moment de discussion, de consultation ou de dispute à l'intérieur d'une interaction conversationnelle considérée comme dominante. Il fonctionnerait «comme dominé par rapport à cette interaction où il apparaîtrait » ${ }^{(12)}$

Dans ce sens, les interactions verbales des émissions «Face à face », qui représentent notre objet d'étude, se caractérisent donc par la coexistence de deux modules : l'interview et la discussion. Reste à savoir lequel des deux dominent les interactions. Nous répondons à cette question dans un autre article. Maintenant, nous allons présenter les caractéristiques de ces deux types de modules. 


\subsubsection{L'interview :}

Le mot « interview » est définit dans le dictionnaire Larousse (2013) comme « un entretient avec une personne pour l'interroger sur ses actes et ses idées, etc. » Donc, il suppose l'existence de deux individus occupant des rôles interlocutifs déterminés par les rôles interactionnels : intervieweur et interviewé. Le premier pose des questions et le second doit y répondre, ayant accepté d'être interviewé. Parlant ainsi à tour de rôle, un transfert d'informations s'effectue entre l'interviewé et l'intervieweur, dans le but de les faire connaitre aux public, pour notre cas il s'agit des téléspectateurs. Ceci implique l'utilisation du dialogue interrogatif, comme le montre l'exemple cidessous.

\section{Exemple :}

5. AJ : voilà messieurs est-ce que vous avez été surpris par cette rupture $\uparrow$ on va dire si rapide de cette fameuse unité nationale dont on imaginait on aurait pu imaginer qu'elle aurait durée $\uparrow$.

6. D1: c'est toujours difficile l'unité nationale surtout lorsque on est à quelques trois semaines moins que trois semaines d'une élection régionale heu bon il y a les les règles de la politique d'opposition est là pour s'opposer etc cela dit c'est vrai que hier c'était assez mal venu c'était trop tôt et dans la forme c'était pas très bien heu bon heu heu la la droite a avait envoyé au front quand même à la fois Christian Istrosi Laurent Voquier Philipe Monier ceux c'est-à-dire qui sont sur la ligne la plus dure on dit que Nicolas Sarcosi les avait un peu remonté ayant trouvé qu'à Versail on avait trop versé dans le consensus j' sais pas si c'est heu voilà si c'est vrai d'un autre coté je trouve que le fait que ce soit Claude Bartelonni qui préside la séance alors que lui-même candidat dans la région de l'ile de France et qui c'est donc espèce de double cascade je ne crois pas que ça soit une très belle idée puisque il manque un peu d'autorité justement pour que dans un cas comme ça pour essayer de faire un rappel à l'ordre///.

7. $\mathrm{AJ}$ : un rappel à l'ordre oui.

8. D1 : résultat effectivement était pas était pas très brillant c'est le moins qu'on puisse dire.

9. AJ : vous pouvez la même chose $\uparrow$ heu heu.

10. D2 : pas tout à fait moi je pense que bon heu lundi il y a eu effectivement l'unité nationale au congrès et c'était le lieu et le moment de le faire parce que le président de la république avait pris la parole et qu'à la fin de cette prise de parole les députés se sont levés et ont chanté la Marseillaise comme vous le disiez après on va pas faire une unité nationale de façade la politique $\operatorname{xxxxx}$ prend ses droits la démocratie ne s'arrête pas et ça serait justement faire un beau cadeau aux terroristes que la politique s'arrête je suis d'accord qu'ils ne se lèvent pas hier c'est peut être heu heu ça fait des images les Français n'aiment pas tellement mais heu en même temps l'opposition a le droit de demander des comptes à la majorité parce que depuis dix mois il y a quand même eu heu des problèmes et en voit bien que heu heu un certain nombre de questions doit être posé alors est-ce qu'il faut 
envoyé les dures heu heu comme il vient de le dire Gérard j'en sais rien mais c'est pas ça qui est///.

Dans cet extrait, il s'agit de deux échanges duels s'opposant à propos d'un désaccord de point de vue. Ce sont des échanges binaires, à chaque fois entre l'animateur-journaliste et l'un de ses deux invités.

Nous constatons qu'il s'agit de la technique de l'interview où les interventions de l'animateur-journaliste sont des questions à double fonction, elles permettent d'une part de sélectionner et mettre en place le locuteur, et d'autre part de contrôler la progression thématique. A travers cette technique, autrement dit l'emploi du couple question/ réponse, l'animateur-journaliste lance le thème tout en sélectionnant un locuteur qui tiendra le rôle de proposant à la ligne (5) et le relance ensuite avec le deuxième locuteur à la ligne (9) ce dernier occupe le rôle d'opposant. Du moment qu'un contrat médiatique les contraint de réagir, les deux antagonistes, eux, y répondent dans le but d'informer et de persuader les téléspectateurs.

Avec ce mode d'allocution, il est difficile de voir une alternance des tours de parole entre les deux débateurs. Les échanges sont duels où l'alternance des tours se fait entre les débateurs et l'animateur-journaliste à tour de rôle, où chacun d'eux expose ses opinions et ses idées. La technique de sélection est egocentrique, elle revient de manière préférentielle à l'animateur-journaliste en raison de l'asymétrie des rôles interlocutifs occupés par les interactants. Dans ce module d'interview, le schéma de l'allocution est le suivant :

Tiers $\longrightarrow$ Proposant $\longrightarrow$ Tiers $\longrightarrow$ pposant

\subsubsection{La discussion :}

La discussion est une forme discursivo-interactionnelle qui se particularise par " une composante argumentative importante » où les partenaires mobilisent toutes leurs ressources linguistiques et stratégiques pour " se convaincre les uns les autres à propos d'un objet de discours particulier. $»^{(13)}$ Elle peut apparaitre dans tous les types d'interactions qu'elles soient formelles (débat médiatique) ou informelles (transaction commerciale). Elle présente certaines caractéristiques de la conversation ordinaire c'est-à-dire une interaction symétrique. Cependant, de par son caractère agonal du à sa visée argumentative elle peut être coopérative (consensuelle) ou conflictuelle (compétitive). Selon Vion, R. ${ }^{(14)}$ « les discussions coopératives orientées vers la recherche d'un consensus et les discussions conflictuelles orientées vers la dispute et l'accentuation des différences.»

A l'inverse de l'interview, dans ce mode d'allocution, l'alternance des tours se fait de manière naturelle, autrement-dit, le contrôle d'allocution n'est pas affecté à une personne précise. Elle se fait selon la technique d'autosélection à des moments de transition possible. Elle se caractérise par la fréquence des interruptions, des intrusions et des chevauchements. Elle apparait généralement pendant le tour d'un débatteur sélectionné par l'animateur- 
journaliste lors de l'émergence d'une situation argumentative à partir d'un désaccord d'opinion.

\section{Exemple :}

15. AJ : j'entends bien alors dans ce cas si j'écoute votre argument pourquoi pleurer sur le lait versé vous auriez dû faire ceci vous auriez dû faire ça c'est pas très constructif pourquoi l'opposition est-ce que dans le fond cette unité nationale ça a gêné l'opposition $\uparrow$ pourtant François Holland a pris des propositions de Nicolas Sarcosi.

16. D1 : ha si ha si si c'est justement c'est justement tout le problème.

17. AJ : oui.

18. D1 : c'est qu'il a clairement quasiment pris toutes les propositions (rires) xxxx par l'opposition par Nicolas Sarcosi.

19. AJ: et même de FN.

20. D1 : donc c'est ce qu'on appelle la triangulation vous prenez les arguments de de votre ///.

21. AJ : la politique de l'adversaire.

22. D1 : l'adversaire et l'adversaire se trouve un/// petit peu dans une situation difficile

23. D2 : oui oui oui mais Gérard je suis je suis d'accord d'ailleurs François Fillon a eu.

24. D1 : rire.

25. D2 : a eu cette formule qui était à mon avis la plus juste de tous ce qu'on a pu entendre à savoir que la plupart des propositions étaient effectivement contenues dans des amendements que la droite avait dans une proposition de loi que la droite avait posée en avril et que la gauche avait refusée de voter donc c'est vrai les choses ils les a dit à sa façon c'est-à-dire calmement sans tomber dans la polémique c'est vrai qu'on sent que les sarcosistes ont été chauffés à blanc si vous voulez ça heu je ça c'est vrai.

26. AJ : oui.

27. D2 : ça c'est vrai et que Alain Jupé en même temps un petit peu faire entendre une petite musique de centre gauche pour faire aussi de la politique tout le monde fait de la politique.

28. AJ : oui.

29. D2 : tout le monde fait de la politique dans cette histoire c'est claire y compris heu heu Claude Bartelonne vous avez raison on peut d'ailleurs s'étonner qu'il préside/// les questionnalités.

30. D1 : les questionnalités.

31. AJ: en faisant $\uparrow$.

32. D2 : après c'est vrai pourquoi ils n'ont pas voté les propositions de

loi du mois d'avril puisque aujourd'hui les reprennent les gens comprennent plus ça les gens comprennent plus ces petits jeux.

33. AJ : mais alors donc vous avez raison la la gauche fait de la triangulation mais surtout la gauche sécurité a pris le pas sur la gauche idéologique c'est du jamais vu c'est la ligne Vals les circonstances//I.

34. D1 : non mais c'est c'est très claire oui mais les circonstances sont tout à fait exceptionnelles et à l'évidence il y avait heu un vrai ///.

35. Aj : En fin on aurait pu imaginer que/// ouî 
36. D1 : sinon un virage en tout cas une inflexion assez forte du président de la république sur heu d'une part sur sa politique étrangère maintenant clairement ///.

37. AJ : oui.

38. D1 : c'est Daeche qui est l'ennemi prioritaire et on plus un peu sur le même plan Deche et Assad et puis sur toutes les mesures sécuritaires qui l'a annoncé hier qui sont $/ / /$.

39. D2 : ce sont $/ / /$.

40. D1 : Je l'aurais dit toutes les mesures qui sont proposées largement par l'opposition c'est un virage très important.

41. D2 : ce sont les inflexions majeurs qui ont réclamées Nicolas Sarcosi puisque l'expression samedi matin c'était ça.

42. AJ : oui.

43. D2 : il faut des $\mathrm{xxxx} \downarrow$.

44. AJ : oui exactement $\downarrow$.

Premièrement, dans cet extrait, nous remarquons qu'il n'y a eu aucune attribution dirigée de la parole par l'animateur-journaliste. La circulation de la parole se fait de manière naturelle autrement dit les deux débatteurs s'autosélectionnent à des moments de transition possible. Ce mode d'allocution est celui de la discussion.

Deuxièmement, si nous nous concentrons sur les tours de parole de 1'animateur-journaliste à qui normalement revient à la fois la gestion de la circulation de la parole ainsi que la progression thématique, nous nous rendrons-compte que de ses 12 tours de parole:

$\checkmark \quad 6$ tours de parole à la ligne (17), (26), (28), (37), (42) et (44) ne sont pas de vrai contribution, ce sont des régulateurs qui servent d'approbation et de suivi aux tours des deux débatteurs ;

$\checkmark \quad 2$ tours de parole à la ligne (19) et (21) sont des complétions c'est-àdire ils servent à compléter et soutenir les tours des deux débatteurs ;

$\checkmark \quad 1$ tour de parole est un acte de langage à la ligne (31) non réussi dans la mesure où il n'a pas eu de réponse ;

$\checkmark \quad 2$ tours de paroles à la ligne (33) et (35) sont des tours interrompus ;

$\checkmark \quad 1$ tour seulement à la ligne (15) qui peut être considéré comme une véritable contribution.

Ceci nous mène à déduire que d'une part les tours de parole qui apportent de vrais contributions à l'activité argumentative sont ceux des deux débatteurs. Cela est tout à fait normal dans la mesure où la position haute leur est attribuée au niveau des rôles interactionnels car ils sont les plus experts dans le domaine. D'autre part, nous remarquons que l'animateur-journaliste à qui revient normalement la gestion de la circulation de la parole abdique la position haute, adopte un comportement en miroir et laisse libre cours à la circulation de la parole entre les deux débatteurs qui se permettent même de léser son territoire en l'interrompant, et en ignorant ces actes de langages. Dans ce module de discussion, le schéma de l'allocution est le suivant :

Tiers $\rightarrow$ proposant $\longrightarrow$ opposant $\longrightarrow$ proposant $\longrightarrow$ opposant 


\title{
4. Synthèse :
}

Les interactions verbales des débats médiatiques télévisés, à vrai dire, les émissions «Face à face» de France 24 qui représentent notre objet d'analyse ne peuvent pas être classées dans un seul type et genre d'interaction particulier. En effet, du point de vue du mode d'allocution, nous avons vu que les trois partenaires à travers leurs activités langagières alternent deux modes de circulation de la parole : l'interview et la discussion qui théoriquement sont deux types d'interaction différents. Dans le premier type, le mode d'allocution est fermé, dirigé par une personne responsable du déroulement interactionnel au niveau de la distribution de la parole ainsi que la progression des thèmes abordés. Dans le deuxième type, le mode d'allocution est ouvert se fait de manière naturelle dans des moments de transition possible. L'alternance de ces deux types d'interaction fait en sorte qu'un réseau de relations interpersonnelles s'instaure entre les partenaires.

\begin{abstract}
Ainsi, inscrire une interaction verbale exclusivement dans un type particulier est une aberration. Traverso V. a écrit à ce sujet : "l'éventail des situations interactionnelles qui ont été étudiées est aujourd'hui immense, montrant le caractère quasiment infini des subdivisions dans ce qui était à un moment considéré comme " un » type d'interaction » ${ }^{(15)} \mathrm{En}$ effet, selon Vion R. ${ }^{(16)}$ :

"Le type n'est pas une image simplifiée de la réalité mais un concept disponible pour l'appréhender. Compte tenu de la complexité et de l'hétérogénéité du réel. " Dans une même rencontre, les protagonistes peuvent mettre en exergue simultanément que successivement, à travers la coordination de leurs actions verbales, deux ou plusieurs types et genres d'interactions selon bien sûr : le but de leur rencontre, la relation qu'ils entretiennent et la coordination de leurs activités discursives.
\end{abstract}

\section{Conclusion:}

L'analyse proposée vise à mettre au clair le classement hétérogène des débats médiatiques et en particulier les émissions télévisées « face à face » de France 24. Cette diversité de classement est due au réseau des relations entretenues par les sujets parlant où nous avons pu repérer une pluralité de positionnement. Cette pluralité ressort de la complexité du cadre participatif qui met en interaction trois participants l'animateur-journaliste et les deux débatteurs. Ces derniers jouant des rôles institutionnels et discursifs différents mettent en place deux espaces relationnels : interlocutif et interactionnel à partir desquels émergent quatre relations différentes qui à leur tour influence et détermine le mode de la circulation de la parole. 


\section{Convention de transcription :}

Pour les conventions de transcription, nous avons utilisé celles de Claire Blanche-

Benveniste (2001) que nous présentons ci-dessous :

- $\uparrow$ : intonation montante.

- $\quad \downarrow$ : intonation descendante.

- $\quad$ xxx : mot inaudible.

- $\quad / / /$ : interruption.

- Chevauchement : chevauchement.

- AJ : animatrice-journaliste.

- D1 : le premier débatteur.

- D2 : le deuxième débatteur.

\section{Note :}

[1] TRAVERSO, V., L'analyse des conversations. Paris: Nathan. P 82. 1999.

[2] VION, R., La communication verbale. Analyse des interactions. Paris: Hachette Supérieur. P124. 1992.

[3 VION, R., L'analyse des interactions verbales. Presses Sorbonne Nouvelle, 1996. 19-32.

[4] KERBRAT-ORECCHIONI, C., \& TRAVERSO, V., Types d'interactions et genres de l'oral. Langages /153, 2004. 41-51.

[5] MAINGUENEAU in KERBRAT-ORECCHIONI, C., \& TRAVERSO, V., Types d'interactions et genres de l'oral. Langages /153, 2004. 41-51.

[6] ANDRE, V. Sociolinguistique des interactions verbales : de l'analyse des situations de travail aux implications sociales 2015. Récupéré sur Revue Langage, Travail et Formation Sociolinguistique des interactions verbales: https://reseaultf.atilf.fr/wp-content/uploads/2015/10/Virgine-Andre.pdf

[7] VION, R., La communication verbale. Analyse des interactions. Paris: Hachette Supérieur, P138. 1992.

[8] PLANTIN, C., Le trilogue argumentatif. Présentation de modèle, analyse de cas. Langue française, $n^{\circ} 112$. L'argumentation en dialogue, sous la direction de Claudine Garcia-Debanc, 1996. 9-30.

[9] PLANTIN, C. (2011). Pour une approche intégrée du champ de l'argumentation: Etat de la question et questions controversées. Dans B.-D. V., Ciências da linguagem e didática das línguas Sciences du langage et didactique des langues: 30 ans de coopération franco-brésilienne 2011. 181-207. Lyon: FAPESP.

[10] Par rapport à ce schéma, le signe « = » exprime une égalité de position et le signe « > » une supériorité de position.

[11] KERBRAT-ORECCHIONI, C., Les interactions verbales, tome I. Paris: A. Colin. P118. 1990.

[12] VION, R. La communication verbale. Analyse des interactions. Paris: Hachette Supérieur. P 149. 1992.

[13] KERBRAT-ORECCHIONI, C. Les interactions verbales, tome I. Paris: A. Colin.pp.118. 1990.

[14] VION, R. (1992). La communication verbale. Analyse des interactions. Paris: Hachette Supérieur. P 137.

[15] TRAVERSO, V., Analyses interactionnelles : repères, questions saillantes et évolution. Dans Langue française $\left(\mathrm{n}^{\circ} 175\right), 2012$. 3-17.

[16] VION, R., L'analyse des interactions verbales. Presses Sorbonne Nouvelle, 1996. 19-32. 